\title{
SQUARE INTEGRABLE DIFFERENTIALS ON RIEMANN SURFACES AND QUASICONFORMAL MAPPINGS
}

\author{
BY
}

\section{CARL DAVID MINDA}

\begin{abstract}
If $f: R \rightarrow R^{\prime}$ is a quasiconformal homeomorphism of Riemann surfaces, then Marden showed that $f$ naturally induces an isomorphism of the corresponding Hilbert spaces of square integrable first-order differential forms. It is demonstrated that this isomorphism preserves many important subspaces. Preliminary to this, various facts about the subspace of semiexact differentials are derived; especially, the orthogonal complement is identified. The norm of the isomorphism is $K(f)^{1 / 2}$ where $K(f)$ is the maximal dilatation of $f$. In addition, $f$ defines an isomorphism of the square integrable harmonic differentials and some important subspaces are preserved. It is shown that not all important subspaces are preserved. The relationship of this to other work is investigated; in particular, the connection with the work of Nakai on the isomorphism of Royden algebras induced by a quasiconformal mapping is explored. Finally, the induced isomorphisms are applied to the classification theory of Riemann surfaces to show that various types of degeneracy are quasiconformally invariant.
\end{abstract}

Introduction. Let $\Gamma(R)$ denote the Hilbert space of square integrable firstorder differential forms on the Riemann surface $R$. A detailed discussion of $\Gamma(R)$ may be found in [3, Chapter V]; free use will be made of the notation and basic results of this reference. Initially, we establish some basic facts about semiexact differentials which are needed later. In particular, the orthogonal complement of the subspace of semiexact differentials is identified and various other orthogonal decompositions are obtained. The main portion of the paper is concerned with the isomorphism $f^{\#}: \Gamma\left(R^{\prime}\right) \rightarrow \Gamma(R)$ induced by a quasiconformal homeomorphism $f: R \rightarrow R^{\prime}$ of Riemann surfaces which was introduced by Marden [6]. A study of $f^{\#}$ shows that it preserves many of the important subspaces and that it has norm $K(f)^{1 / 2}$, where $K(f)$ is the maximal dilatation of $f$. Also, a quasiconformal mapping $f: R \rightarrow R^{\prime}$ induces an isomorphism $f_{b}^{\#}: \Gamma_{b}\left(R^{\prime}\right) \rightarrow \Gamma_{b}(R)$ of square integrable harmonic differentials. Again, many important subclasses of

Received by the editors October 19, 1972 and, in revised form, July 17, 1973 and October 15, 1973.

AMS (MOS) subject classifications (1970). Primary 30A52; Secondary 30A48, 30A60.

Key words and phrases. Square integrable differential forms, semiexact differentials, classification theory, quasiconformal mappings, isomorphisms of differentials. 
harmonic differentials are preserved under this isomorphism; however, several subspaces are exhibited which are not preserved. Nakai [11] showed that a quasiconformal mapping of Riemann surfaces naturally induces an isomorphism between the corresponding Royden algebras and that the converse is also true. The relationship of this to the isomorphism of Marden is investigated. The connection with work of Rochberg ([13], [14]) and Accola [2] is discussed, too. Finally, some applications to the classification theory of Riemann surfaces are given which demonstrate the quasiconformal invariance of several null classes.

1. Semiexact differentials and orthogonal decompositions. In this section we shall discuss the subspace of semiexact differentials, identify its orthogonal complement and derive several other related orthogonal decompositions. All of these results will be employed later.

Definition. Let $R$ be a Riemann surface.

(i) $\Gamma_{s e}^{1}(R)=\left\{\omega \in \Gamma^{1}(R): \int_{d} \omega=0\right.$ for all dividing cycles $\left.d\right\} . \Gamma_{s e}(R)$ is the closure of $\Gamma_{s e}^{1}(R)$ in the Hilbert space $\Gamma(R)$.

(ii) $\Gamma_{c o e}(R)$ is the closure of $\Gamma_{c o}^{1}(R) \cap \Gamma_{e}^{1}(R)$ in $\Gamma(R)$.

From the definition it is clear that $\Gamma_{c}^{1} \supset \Gamma_{s e}^{1} \supset \Gamma_{c o}^{1}, \Gamma_{e}^{1}$; consequently, $\Gamma_{c} \supset$ $\Gamma_{s e} \supset \Gamma_{c o}, \Gamma_{e}$. Because $\Gamma_{e o}^{1} \subset \Gamma_{c o}^{1} \cap \Gamma_{e}^{1} \subset \Gamma_{c o} \cap \Gamma_{e}$, it is evident that $\Gamma_{e o} \subset$ $\Gamma_{c o e} \subset \Gamma_{c o} \cap \Gamma_{e}$

Proposition 1. $\Gamma_{s e}(R) \cap \Gamma^{1}(R)=\Gamma_{s e}^{1}(R)$.

Proof. Obviously, $\Gamma_{s e}^{1} \subset \Gamma_{s e} \cap \Gamma^{1}$. Now let $\omega \in \Gamma_{s e} \cap \Gamma^{1} \subset \Gamma_{c} \cap \Gamma^{1}=\Gamma_{c}^{1}$. There is a sequence $\left(\omega_{n}\right)_{n=0}^{\infty}$ in $\Gamma_{\text {se }}^{1}$ with $\left\|\omega-\omega_{n}\right\| \rightarrow 0$. Let $d$ be any dividing cycle on $R$, then $\int_{d} \omega_{n}=0$ for all $n$. Since $\sigma \rightarrow \int_{d} \sigma$ is a continuous linear functional on $\Gamma_{c}^{1}$, it follows that $\int_{d} \omega=0$. Hence, $\omega \in \Gamma_{s e}^{1}$.

Corollary. $\Gamma_{b s e}(R)=\Gamma_{s e}(R) \cap \Gamma_{b}(R)$

The next result gives several useful orthogonal decompositions involving $\Gamma_{s e^{\bullet}}$

Proposition 2. (i) $\Gamma_{s e}(R)=\Gamma_{c o}(R) \oplus\left(\Gamma_{b s e}(R) \cap \Gamma_{b e}^{*}(R)\right)$.

(ii) $\Gamma_{s e}(R)=\Gamma_{b s e}(R) \oplus \Gamma_{e o}(R)$.

(iii) $\Gamma_{c}(R)=\Gamma_{s e}(R) \oplus \Gamma_{b m}^{*}(R)$.

Proof. (i) This follows in a simple manner from the decomposition $\Gamma=\Gamma_{\text {co }}$ $\oplus \Gamma_{e}^{*}$. Let $\omega \in \Gamma_{s e}$, then $\omega=\omega_{c o}+{ }^{*} \omega_{e}$ where $\omega_{c o} \in \Gamma_{c o}$ and $\omega_{e} \in \Gamma_{e}$. Since $\omega_{c o} \in \Gamma_{c o} \subset \Gamma_{s e}$, 


$$
{ }^{*} \omega_{e} \in \Gamma_{s e} \cap \Gamma_{e}^{*}=\Gamma_{s e} \cap \Gamma_{c} \cap \Gamma_{c}^{*} \cap \Gamma_{e}^{*}=\Gamma_{b s e} \cap \Gamma_{b e}^{*}
$$

(ii) The result is obtained immediately upon applying the orthogonal decomposition $\Gamma_{c}=\Gamma_{b} \oplus \Gamma_{e o}$ to a differential $\omega \in \Gamma_{\text {se }} \subset \Gamma_{c}$.

(iii) This decomposition is derived from (ii) together with $\Gamma_{c}=\Gamma_{b} \oplus \Gamma_{e o}=$ $\Gamma_{b m}^{*} \oplus \Gamma_{b s e} \oplus \Gamma_{e o^{\circ}}$

The following is a slight extension of a theorem of Royden ([16, p. 53], [17, Theorem 13B, p. 134]).

Theorem 1. $\Gamma_{e}(R)=\Gamma_{c o e}(R) \oplus\left(\Gamma_{b s e}^{*}(R) \cap \Gamma_{b e}(R)\right)$.

Proof. First we show that $\Gamma_{c o e} \perp \Gamma_{b s e}^{*} \cap \Gamma_{b e^{*}}$ Actually it is enough to prove that $\Gamma_{c o}^{1} \cap \Gamma_{e}^{1} \perp \Gamma_{b s e}^{*} \cap \Gamma_{b e}$. Let $d f \in \Gamma_{c o}^{1} \cap \Gamma_{e}^{1}$ and $d u \in \Gamma_{b s e}^{*} \cap \Gamma_{b e}$. Determine a canonical region $\Omega \subset R$ such that $d f=0$ in $R \sim \Omega$. Let $\partial \Omega=\bigcup \gamma_{n}$ where each contour $\gamma_{n}$ is positively oriented. $f$ is constant on each $\gamma_{n}$, say $f \mid \gamma_{n}=c_{n}$. Also, each $\gamma_{n}$ is a dividing cycle because $\Omega$ is canonical. Now,

$$
\begin{aligned}
(d f, d u)_{R} & =(d f, d u)_{\Omega}=\iint_{\mathbb{Q}} d f \wedge^{*} d \bar{u} \\
& =\int_{\partial \Omega} f * d \bar{u}-\iint_{\Omega} f d * d \bar{u}=\sum_{n} c_{n} \int_{\gamma_{n}} * d \bar{u}-\iint_{\Omega} f d * d \bar{u} .
\end{aligned}
$$

Since $d \bar{u} \in \Gamma_{b e}, d^{*} d \bar{u}=\Delta \bar{u}=0$. As $d \bar{u} \in \Gamma_{b s e}^{*}$ and $\gamma_{n}$ is a dividing cycle, $\int_{\gamma_{n}} * d \bar{u}=0$. Hence, $(d f, d u)_{R}=0$. Next, if $\omega \in \Gamma_{e}$ and $\omega \perp \Gamma_{\text {coe }}$, then $\omega \in \Gamma_{b \text { se }}^{*}$ $\cap \Gamma_{b e^{\cdot}}$ Since $\Gamma_{c o e} \supset \Gamma_{e o^{\circ}} \omega \perp \Gamma_{e o^{\circ}}$ From $\Gamma=\Gamma_{e o} \oplus \Gamma_{c}^{*}$ we obtain $\omega \in \Gamma_{e} \cap$ $\Gamma_{c}^{*}=\Gamma_{b e^{\cdot}}$ It remains to show that $\omega \in \Gamma_{b s e^{*}}^{*}$ Because $\omega \in \Gamma_{b e}, \omega=d u$ for some harmonic function $u$ on $R$ having finite Dirichlet integral. Let $d$ be any dividing cycle on $R$. Take a regular open set $\Omega$ on $R$ such that $\partial \Omega=d \cup d^{\prime}$ with $d^{\prime}$ homologous to $d$. It may be necessary to replace $d$ by a cycle homologous to $d$ in order to accomplish this but no generality is lost. There is a smooth function $g$ on $R$ with $g=1$ on $d, g=0$ on $d^{\prime}$ and $d g=0$ on $R \sim \Omega$. Then $d g \in \Gamma_{c o}^{1} \cap \Gamma_{e}^{1}$ and

$$
0=(d g, d u)_{R}=(d g, d u)_{Q}=\iint_{\mathbb{Q}} d g \wedge * d \bar{u}=\int_{\partial \Omega} g^{*} d \bar{u}=\int_{d} * d \bar{u}
$$

Thus, ${ }^{*} d \bar{u}$ is semiexact so ${ }^{*} d u$ is too. Therefore, $\omega=d u \in \Gamma_{b s e}^{*} \cap \Gamma_{b e^{*}}$

Corollary. $\Gamma(R)=\Gamma_{\text {se }}^{*}(R) \oplus \Gamma_{\text {coe }}(R)=\Gamma_{\text {se }}(R) \oplus \Gamma_{\text {coe }}^{*}(R)$.

Proof. From the theorem $\Gamma_{e}=\Gamma_{c o e} \oplus \Gamma_{b s e}^{*} \cap \Gamma_{b e}$ and it is known that $\Gamma=$ 
$\Gamma_{c o}^{*} \oplus \Gamma_{e}$ so that $\Gamma=\Gamma_{c o}^{*} \oplus\left(\Gamma_{b s e}^{*} \cap \Gamma_{b e}\right) \oplus \Gamma_{c o e}$. Proposition 2 (i) gives $\Gamma_{\text {se }}=$ $\Gamma_{c o} \oplus \Gamma_{b s e} \cap \Gamma_{b e}^{*}$ so the corollary now follows.

Corollary. $\Gamma_{c o e}(R)=\Gamma_{e o}(R) \oplus \Gamma_{b m}(R)$.

Proof. Recall that $\Gamma_{e}=\Gamma_{b e} \oplus \Gamma_{e o}$ and $\Gamma_{b e}=\Gamma_{b m} \oplus\left(\Gamma_{b s e}^{*} \cap \Gamma_{b e}\right)$ so that $\Gamma_{e}=\Gamma_{e o} \oplus \Gamma_{b m} \oplus\left(\Gamma_{b s e}^{*} \cap \Gamma_{b e}\right)$. By comparing this decomposition of $\Gamma_{e}$ to that given in the theorem and using the uniqueness of an orthogonal complement, we obtain the corollary.

The first corollary proves that $\Gamma_{\text {coe }}^{*}(R)$ and $\Gamma_{\text {se }}(R)$ are orthogonal complements in $\Gamma(R)$. Since $\Gamma=\Gamma_{e o} \oplus \Gamma_{c}^{*}$, a consequence of the second corollary is that $\Gamma_{c o e} \cap \Gamma_{c}^{*}=\Gamma_{b m}$. Clearly, $\Gamma_{c o} \cap \Gamma_{e} \cap \Gamma_{c}^{*}=\Gamma_{b o} \cap \Gamma_{b e^{*}}$ In general, $\Gamma_{b m}$ is a proper subset of $\Gamma_{b o} \cap \Gamma_{b e}[1]$, so $\Gamma_{c o e}$ is typically properly included in $\Gamma_{c o}$ $\cap \Gamma_{e^{\bullet}}$

2. Isomorphism of square integrable differentials induced by a quasiconformal mapping. Suppose that $f: R \rightarrow R^{\prime}$ is a $K$-quasiconformal mapping of the Riemann surface $R$ onto the Riemann surface $R^{\prime}$, then $f$ induces an isomorphism $f^{\#}: \Gamma\left(R^{\prime}\right)$ $\rightarrow \Gamma(R)$ as follows $[6, \mathrm{p} .30]$. Let $\omega^{\prime}=a(w) d u+b(w) d v$ in terms of a local parameter $w=u+i v$ in a neighborhood of $p^{\prime}=f(p)$, then $f^{\#}\left(\omega^{\prime}\right)$ is defined in a neighborhood of $p$ by

$$
f^{\#}\left(\omega^{\prime}\right)=\left[a(f(z)) u_{x}(z)+b(f(z)) v_{x}(z)\right] d x+\left[d(f(z)) u_{y}(z)+b(f(z)) v_{y}(z)\right] d y
$$

in terms of the local parameter $z=x+i y$ at $p$ where $f=u+i v$. Here $u_{x}, u_{y}, v_{x^{\prime}}$, $v_{y}$ are distributional derivatives of $u$ and $v$. Proofs of several properties of $f^{*}$ are sketched in [6]; complete proofs are included here together with many new properties of $f^{\#}$.

Theorem 2. (i) If $f: R \rightarrow R^{\prime}$ is $K$-quasiconformal, then

$$
f^{\#}\left(a \omega^{\prime}+b \sigma^{\prime}\right)=a f^{\#}\left(\omega^{\prime}\right)+b f^{\#}\left(\sigma^{\prime}\right)
$$

for all $a, b \in \mathbf{C}$ and $\omega^{\prime}, \sigma^{\prime} \in \Gamma\left(R^{\prime}\right)$ and

$$
K^{-1 / 2}\left\|\omega^{\prime}\right\|_{R^{\prime}} \leq\left\|f^{\#}\left(\omega^{\prime}\right)\right\|_{R} \leq K^{1 / 2}\left\|\omega^{\prime}\right\|_{R^{\prime}}
$$

(ii) If $j: R \rightarrow R$ is the identity mapping, then $j^{\#}: \Gamma(R) \rightarrow \Gamma(R)$ is the identity mapping.

(iii) If $f: R \rightarrow R^{\prime}$ and. $g: R^{\prime} \rightarrow R^{\prime \prime}$ are quasiconformal bomeomorphisms, then $(g \circ f)^{\#}=f^{\#} \circ g^{\#}$.

Proof. (i) That $f^{\#}$ is linear is clear. It is sufficient to establish the righthand inequality, since the other inequality then follows by considering $f^{-1}: R^{\prime} \rightarrow R$ 
which is also $K$-quasiconformal and making use of properties (ii) and (iii). The right-hand inequality shows that $f^{\prime \prime}$ maps $\Gamma\left(R^{\prime}\right)$ into $\Gamma(R)$. In fact, it is enough to show that $\left\|f^{\sharp}\left(\omega^{\prime}\right)\right\|_{V}^{2} \leq K\left\|\omega^{\prime}\right\|_{V^{\prime}}^{2}$ where $V$ is a Jordan region on $R$ and $V^{\prime}=f(V)$. Suppose $\omega^{\prime}=a(w) d u+b(w) d v$ in $V^{\prime}$. Let

$$
D f(z)=\left[\begin{array}{ll}
u_{x}(z) & u_{y}(z) \\
v_{x}(z) & v_{y}(z)
\end{array}\right]
$$

which is the matrix representing the derivative of $f$ at $z \in V . D f(z)$ exists a.e. since $f$ is quasiconformal. Now,

$$
D f(z)^{\operatorname{tr}}\left[\begin{array}{l}
d(f(z)) \\
b(f(z))
\end{array}\right]=\left[\begin{array}{l}
d(f(z)) u_{x}(z)+b(f(z)) v_{x}(z) \\
d(f(z)) u_{y}(z)+b(f(z)) v_{y}(z)
\end{array}\right],
$$

where $D f(z)^{\text {tr }}$ denotes the transpose of the matrix $D f(z)$. Let $\|D f(z)\|$ denote the norm of the linear transformation $D f(z): \mathbf{R}^{2} \rightarrow \mathbf{R}^{2}$, then $\|D f(z)\|^{2} \leq K|J(z, f)|$ a.e. where $J(z, f)$ is the Jacobian of $f$ at $z$ [18, Theorem 34.6, p. 115]. By making use of this fact together with (1) we obtain

$$
\begin{aligned}
\left\|f^{\#}\left(\omega^{\prime}\right)\right\|_{V}^{2} & \leq K \iint_{V}\left(|d(f(z))|^{2}+|b(f(z))|^{2}\right)|J(z, f)| d x d y \\
& =K \iint_{f(V)=V^{\prime}}\left(|d(w)|^{2}+|b(w)|^{2}\right) d u d \nu=K\left\|\omega^{\prime}\right\|_{V^{\prime \bullet}}^{2}
\end{aligned}
$$

(ii) and (iii) are trivial to verify.

Corollary. If $f: R \rightarrow R^{\prime}$ is quasiconformal, then $f^{\#}: \Gamma\left(R^{\prime}\right) \rightarrow \Gamma(R)$ is an isomorpbism of Hilbert spaces.

Theorem 3. If $f: R \rightarrow R^{\prime}$ is a quasiconformal bomeomorphism, then the isomorphism $f^{\#}: \Gamma\left(R^{\prime}\right) \rightarrow \Gamma(R)$ is also an isomorphism of

onto

$$
\Gamma_{c}\left(R^{\prime}\right), \Gamma_{s e}\left(R^{\prime}\right), \Gamma_{e}\left(R^{\prime}\right), \Gamma_{c o}\left(R^{\prime}\right), \Gamma_{c o e}\left(R^{\prime}\right), \Gamma_{e o}\left(R^{\prime}\right)
$$

$$
\Gamma_{c}(R), \Gamma_{s e}(R), \Gamma_{e}(R), \Gamma_{c o}(R), \Gamma_{c o e}(R), \Gamma_{e o}(R),
$$

respectively.

Proof. The cases corresponding to $\Gamma_{c}, \Gamma_{e}$ and $\Gamma_{e o}$ were already mentioned by Marden [6, p. 30]. The proof of these facts is scattered throughout the remainder 
of this section. The next three propositions will be used in establishing this theorem.

Proposition 3. Suppose $R$ is a Riemann surface, $b: R \rightarrow \mathrm{C}$ is continuous and bas locally integrable distributional derivatives. If $\sigma \in \Gamma_{c}^{1}(R)$ and $\Omega$ is a regular subregion on $R$, then $\iint_{\Omega} d b \wedge \sigma=\int_{\partial \Omega} b \sigma$.

Proof. Let $\left(\Delta_{n}\right)_{n=0}^{N}$ be a triangulation of $\bar{\Omega}$ such that $\Delta_{n}$ is a compact subset of a parametric disk $V_{n}$ on $R$. Since $\sigma \in \Gamma_{c}^{l}(R)$, there is a twice continuously differentiable function $g_{n}: V_{n} \rightarrow \mathrm{C}$ with $d g_{n}=\sigma \mid V_{n}$. Hence,

$$
\iint_{\mathbb{Q}} d b \wedge \sigma=\sum_{n=0}^{N} \iint_{\Delta_{n}} d b \wedge d g_{n}=\sum_{n=0}^{N} \iint_{\Delta_{n}}\left(\frac{\partial b}{\partial x} \frac{\partial g_{n}}{\partial y}-\frac{\partial b}{\partial y} \frac{\partial g_{n}}{\partial x}\right) d x d y \text {. }
$$

Now,

$$
\iint_{\Delta_{n}}\left(\frac{\partial b}{\partial x} \frac{\partial g_{n}}{\partial y}-\frac{\partial b}{\partial y} \frac{\partial g_{n}}{\partial x}\right) d x d y=\int_{\partial \Delta_{n}} b d g_{n}=\int_{\partial \Delta_{n}} b \omega
$$

[5, p. 156]. Since $\sum_{n=0}^{N} \int_{\partial \Delta_{n}} b \omega=\int_{\partial Q} b \omega$, the result follows.

Proposition 4. Suppose $R$ is a Riemann surface and $\sigma \in \Gamma(R)$. If for every parametric disk $V$ on $R, \sigma \mid V \in \Gamma_{c}(V)$, then $\sigma \in \Gamma_{c}(R)$.

Proof. It is sufficient to show that $\sigma \perp \Gamma_{\text {eo }}^{*}$ since $\Gamma=\Gamma_{c} \oplus \Gamma_{e 0}^{*}$. In fact, it is enough to prove that $\sigma \perp \Gamma_{e 0^{\circ}}^{1 *}$ Let $d b \in \Gamma_{e o}^{1}$ and let $K$ be a compact set such that $b$ vanishes on $R \sim K$. Find a finite set $\left(V_{n}\right)_{n=0}^{N}$ of parametric disks $V_{n}$ such that $K \subset \bigcup_{n=0}^{N} V_{n}$. By selecting the $V_{n}$ properly it is possible to find $d b_{n} \epsilon$ $\Gamma_{e o}^{1}\left(V_{n}\right)$ such that $\Sigma_{n=0}^{N} b_{n} \equiv 1$ on $K$. Now, $b b_{n} \in \Gamma_{e o}^{1}\left(V_{n}\right)(0 \leq n \leq N)$ and since $\sigma \mid V_{n} \in \Gamma_{c}\left(V_{n}\right)$, we obtain $0=\left(\sigma, * d\left(b b_{n}\right)\right)_{V_{n}}=\left(\sigma, * d\left(b b_{n}\right)\right)_{R}$. By summing this over $n$ we get $(\sigma, * d b)_{R}=0$. Hence, $\sigma \in \Gamma_{c}(R)$.

Proposition 5. Suppose $R$ is a Riemann surface and $\sigma \in \Gamma_{c}(R)$. If $\sigma$ bas compact support, then $\sigma \in \Gamma_{c o}(R)$.

Proof. We will show that $\sigma \perp \Gamma_{e}^{1 *}$; the result then follows since $\Gamma=\Gamma_{c o} \oplus$ $\Gamma_{e}^{*}$. Take $d b \in \Gamma_{e}^{1}$ and let $\Omega$ be a regular region on $R$ such that $\omega=0$ in $R \sim \Omega$. Let $g: R \rightarrow \mathrm{C}$ be twice continuously differentiable such that $g$ has compact support and $g \mid \Omega=1$. Then $d g \in \Gamma_{e o}^{1}$ and $\left.d g b\right) \in \Gamma_{e 0^{\circ}}^{1}$ Since $\sigma \in \Gamma_{c}$ and $\Gamma_{c} \perp \Gamma_{e 0}^{*}$, it follows that $0=(\sigma, * d(g b))_{R}=(\sigma, * d(g b))_{Q}=(\sigma, * d b)_{Q}=(\sigma, * d b)_{R}$. Thus, $\sigma \in \Gamma_{\text {co }}(R)$.

Now we are in a position to show that $f^{\#}$ preserves $\Gamma_{c}$. We will prove $f^{\#}\left(\Gamma_{c}^{1}\left(R^{\prime}\right)\right)$ is a subset of $\Gamma_{c}(R)$. From the continuity of $f^{\sharp}$ it will follow that 
$f^{\#}\left(\Gamma_{c}\left(R^{\prime}\right)\right) \subset \Gamma_{c}(R)$. Consideration of $f^{-1}$ shows that equality must hold. Let $\omega^{\prime} \in \Gamma_{c}^{1}\left(R^{\prime}\right)$; it is enough to prove that $f^{\sharp}\left(\omega^{\prime}\right)$ is locally closed on $R$. Let $V$ be a parametric disk on $R$ and set $V^{\prime}=f(V)$. Since $\omega^{\prime} \in \Gamma_{c}^{l}\left(R^{\prime}\right)$, there is a twice continuously differentiable function $g: V^{\prime} \rightarrow \mathrm{C}$ with $d g=\omega^{\prime}$ in $V^{\prime}$. It is a simple matter to see that $f^{\#}\left(\omega^{\prime}\right) \mid V=d(g \circ f)$. We want to demonstrate that $d(g \circ f) \perp$ $\Gamma_{e o}^{1}(V)$. Let $d b \in \Gamma_{e o}^{1}(V)$ and $\Delta$ be a Jordan domain in $V, \bar{\Delta} c V$, such that $\partial \Delta$ is rectifiable and $b \equiv 0$ in $V \sim \Delta$, then by Proposition 3

$$
\left(d(g \circ f),{ }^{*} d h\right)_{V}=\iint_{\Delta} d \bar{b} \wedge d(g \circ f)=\int_{\partial \Delta} \bar{b} d(g \circ f)=0
$$

since $\bar{b}=0$ on $\partial \Delta$. Thus, $f^{\#}$ is an isomorphism of $\Gamma_{c}\left(R^{\prime}\right)$ onto $\Gamma_{c}(R)$.

Next, $f^{\#}\left(\Gamma_{c o}\left(R^{\prime}\right)\right)=\Gamma_{c o}(R)$. We will prove that $f^{\#}\left(\Gamma_{c o}^{1}\left(R^{\prime}\right)\right) \subset \Gamma_{c o}(R)$ which suffices. Let $\omega^{\prime} \in \Gamma_{c o}^{1}\left(R^{\prime}\right) \subset \Gamma_{c}\left(R^{\prime}\right)$, then $f^{\#}\left(\omega^{\prime}\right) \in \Gamma_{c}(R)$ and has compact support. From Proposition 5 we conclude that $f^{\#}\left(\omega^{\prime}\right) \in \Gamma_{c o}(R)$.

$f^{\#}$ is an isomorphism of $\Gamma_{\text {coe }}\left(R^{\prime}\right)$ onto $\Gamma_{\text {coe }}(R)$. As is typical we will just show that $f^{\#}$ maps $\Gamma_{c o}^{1}\left(R^{\prime}\right) \cap \Gamma_{e}^{1}\left(R^{\prime}\right)$ into $\Gamma_{c o e}(R)$. Let $d g \in \Gamma_{c o}^{1}\left(R^{\prime}\right) \cap \Gamma_{e}^{1}\left(R^{\prime}\right)$, then $f^{\#}(d g)=d(g \circ f)$. Note that $g \circ f$ is continuous on $R$, has compact support and has square integrable distributional derivatives. Let $\sigma \in \Gamma_{s e}^{1}(R) \subset \Gamma_{c}^{1}(R)$ and take $\Omega$ to be a canonical regular region on $R$ outside of which $d(g \circ f)$ vanishes, then by Proposition 3

$$
\left(d(g \circ f),{ }^{*} \sigma\right)_{R}=\left(d(g \circ f),{ }^{*} \sigma\right)_{\Omega}=-\iint_{\Omega} d(g \circ f) \wedge \bar{\sigma}=-\int_{\partial \Omega}(g \circ f) \bar{\sigma} .
$$

Since $g \circ f$ is constant on each contour of $\partial \Omega$, each contour of $\partial \Omega$ is a dividing cycle and $\sigma \in \Gamma_{s e}^{1}(R)$, it follows that the last integral vanishes. Thus, $d(g \circ f) \perp$ $\Gamma_{\text {se }}^{*}(R)$ and so $d(g \circ f) \in \Gamma_{\text {coe }}(R)$.

By a very similar type of argument one can show that $f^{\#}$ is an isomorphism of $\Gamma_{e}\left(R^{\prime}\right), \Gamma_{e o}\left(R^{\prime}\right)$ onto $\Gamma_{e}(R), \Gamma_{e o}(R)$, respectively. For this reason the proofs will not be given. The next proposition is useful in studying the isomorphism between continuous linear functionals on $\Gamma(R)$ and $\Gamma\left(R^{\prime}\right)$ which is induced by $f^{\#}$. In turn this will enable us to show that $f^{\#}$ maps $\Gamma_{s e}\left(R^{\prime}\right)$ onto $\Gamma_{s e}(R)$.

Proposition 6. Suppose that $f: R \rightarrow R^{\prime}$ is a K-quasiconformal bomeomorphism, $f_{n}: R \rightarrow R_{n}^{\prime} \subset R^{\prime}$ is a K-quasiconformal bomeomorphism, $\left(f_{n}\right)_{n=0}^{\infty}$ converges uniformly to $f$ on compact subsets of $R$ and $\left\|d f-d f_{n}\right\|_{K} \rightarrow 0$ for some compact set $K \subset R$. For $\omega^{\prime} \in \Gamma\left(R^{\prime}\right)$ let $f_{n}^{\#}\left(\omega^{\prime}\right)$ denote the image of $\omega^{\prime} \mid R_{n}^{\prime}$ under $f_{n}^{\#}$, then $\left\|f^{\#}\left(\omega^{\prime}\right)-f_{n}^{\#}\left(\omega^{\prime}\right)\right\|_{K} \rightarrow 0$ for all $\omega^{\prime} \in \Gamma\left(R^{\prime}\right)$.

Proof. First, an elementary argument shows that we need only establish the result for $\omega^{\prime} \in \Gamma^{1}\left(R^{\prime}\right)$ and when $K$ is a compact subset of some parametric disk $V$. In the following we make the abuse of notation that $f^{\#}\left(\omega^{\prime}\right)$ also represents a 
$2 \times 1$ column vector whose top component is the coefficient of $d x$ and whose bottom component is the coefficient of $d y$. With this convention

$$
\begin{aligned}
& f^{H}(\omega)-f_{n}^{\#}(\omega)=D f(z)^{\mathrm{tr}}\left[\begin{array}{l}
d(f(z)) \\
b(f(z))
\end{array}\right]-D f_{n}(z)^{\mathrm{tr}}\left[\begin{array}{l}
d\left(f_{n}(z)\right) \\
b\left(f_{n}(z)\right)
\end{array}\right] \\
& =D f(z)^{\mathrm{tr}}\left[\begin{array}{l}
\left.d(f(z))-d\left(f_{n}(z)\right)\right) \\
b(f(z))-b\left(f_{n}(z)\right)
\end{array}\right]-D\left(f(z)-f_{n}(z)\right)^{\mathrm{tr}}\left[\begin{array}{l}
\left.d\left(f_{n}(z)\right)\right) \\
b\left(f_{n}(z)\right)
\end{array}\right],
\end{aligned}
$$

so that

$$
\left\|f^{\#}\left(\omega^{\prime}\right)-f_{n}^{\#}\left(\omega^{\prime}\right)\right\| \leq\|D f(z)\|\left\|\begin{array}{l}
a(f(z))-a\left(f_{n}(z)\right) \\
b(f(z))-b\left(f_{n}(z)\right)
\end{array}\right\|+\left\|D\left(f(z)-f_{n}(z)\right)\right\|\left\|\begin{array}{l}
d\left(f_{n}(z)\right) \\
b\left(f_{n}(z)\right)
\end{array}\right\| .
$$

There is a compact set $K^{\prime} \subset R^{\prime}$ such that $f(K) \subset K^{\prime}$ and $f_{n}(K) \subset K^{\prime}$ for all $n$ sufficiently large. Since $a$ and $b$ are continuous on $K^{\prime}$, it follows that $a \circ f_{n}$ and $b \circ f_{n}$ are uniformly bounded on $K$. Also, since $a$ and $b$ are uniformly continuous on $K^{\prime}$ and $\left(f_{n}\right)_{n=0}^{\infty}$ converges uniformly to $f$ on $K$, it is clear that $a$ of $a \circ f_{n}$ and $b \circ f-b \circ f_{n}$ converge uniformly to zero on $K$. Finally,

$$
\left\|D\left(f(z)-f_{n}(z)\right)\right\| \leq\left|\frac{\partial\left(f-f_{n}\right)}{\partial x}(z)\right|+\left|\frac{\partial\left(f-f_{n}\right)}{\partial y}(z)\right| .
$$

These remarks in conjunction with inequality (2) are satisfactory to indicate the method of completing the proof. Therefore, the proof is complete.

Since $f^{\#}$ is an isomorphism of $\Gamma_{c}\left(R^{\prime}\right)$ onto $\Gamma_{c}(R)$, it is well known that $f^{*}$ induces an isomorphism between the continuous linear functionals on $\Gamma_{c}(R)$ and those on $\Gamma_{c}\left(R^{\prime}\right)$. In fact, if $L: \Gamma_{c}(R) \rightarrow \mathrm{C}$ is a continuous linear functional on $\Gamma_{c}(R)$, then the mapping $L^{\prime}: \Gamma_{c}\left(R^{\prime}\right) \rightarrow \mathrm{C}$ defined by $L^{\prime}\left(\omega^{\prime}\right)=L\left(f^{\prime \prime}\left(\omega^{\prime}\right)\right)$ is the corresponding linear functional on $\Gamma_{c}\left(R^{\prime}\right)$. We shall investigate this connection more closely in a special case. Let $c$ be a 1-cycle on $R$, then $L(\omega)=\int_{c} \omega$ is a continuous linear functional on $\Gamma_{c}^{1}(R)[3, \mathrm{pp} .284-286]$ which has a unique extension to $\Gamma_{c}(R)$. The extension is still denoted by $L$. By the Riesz representation theorem there is a unique differential $\psi(c) \in \Gamma_{c}(R)$ such that $L(\omega)=$ $(\omega, \psi(c))_{R}$ for all $\omega \in \Gamma_{c}(R)$. Note that $\psi(c)$ depends only on the homology class of c. Also, since $\Gamma_{c}(R)=\Gamma_{e o}(R) \oplus \Gamma_{b o}^{*}(R)$, it follows that $\psi(c) \in \Gamma_{b o}^{*}(R)$. The corresponding linear functional on $\Gamma_{c}\left(R^{\prime}\right)$ is $L^{\prime}\left(\omega^{\prime}\right)=\left(f^{\#}\left(\omega^{\prime}\right), \psi(c)\right)_{R}$. The following theorem shows that $L^{\prime}$ is simply the linear functional naturally associated with the cycle $c^{\prime}=f(c)$; see $[6, \mathrm{pp} .30-31]$. Let $\psi^{\prime}\left(c^{\prime}\right)$ be the differential associated with $c^{\prime}$. 
Theorem 4. Let $f: R \rightarrow R^{\prime}$ be a K-quasiconformal bomeomorpbism. If $c$ is a 1-cycle on $R$ and $c^{\prime}=f(c)$, then $\left(\omega^{\prime}, \psi^{\prime}\left(c^{\prime}\right)\right)_{R^{\prime}}=\left(f^{\prime \prime}\left(\omega^{\prime}\right), \psi(c)\right)_{R}$ for all $\omega^{\prime} \epsilon$ $\Gamma_{c}\left(R^{\prime}\right)$.

Proof. First, observe that it suffices to establish the result in the case when $\omega^{\prime} \in \Gamma_{c}^{1}(R)$ and $c$ is a smooth Jordan. curve on $R$. Let $A$ be an annular neighborhood about $c$, then $f(A)=A^{\prime}$ is an annular region on $R^{\prime}$. Take an annular neighborhood $A_{1}$ of $c$ with $\bar{A}_{1} \subset A$. There is a sequence $\left(f_{n}\right)_{n=0}^{\infty}$ of real-analytic $K$ quasiconformal maps of $A_{1}$ into $A^{\prime}$ such that $\left(f_{n}\right)_{n=0}^{\infty}$ converges uniformly to $f$ on compact subsets of $A_{1}$ [5, Satz 4.2, p. 222] and $\left\|d f-d f_{n}\right\|_{K} \rightarrow 0$ for all compact sets $K \subset A_{1}$ [5, Satz 5.3, p. 227]. Let $\omega^{\prime} \in \Gamma_{c}^{1}\left(R^{\prime}\right)$. A straightforward computation shows that

$$
\int_{c} f_{n}^{\prime \prime}\left(\omega^{\prime}\right)=\int_{f_{n}(c)} \omega^{\prime}=\int_{c^{\prime}} \omega^{\prime}=\left(\omega^{\prime}, \psi^{\prime}\left(c^{\prime}\right)\right)_{R^{\prime \prime}}
$$

since $f_{n}(c)$ is homologous to $f(c)=c^{\prime}$, at least for sufficiently large values of $n$. Let $\sigma$ be a closed differential having compact support $K, \subset \subset K \subset A_{1}$, such that

$$
\int_{c} r=\iint_{K} \sigma \wedge \tau=\left(\tau, *^{*}\right)_{R}=\left(\tau, *^{*}\right)_{K}
$$

for all $r \in \Gamma_{c}^{1}(R)$ [4, pp. 283-285]. This remains valid for any $r \in \Gamma_{c}^{1}(\Omega)$ where $\Omega$ is a subregion of $R$ which contains $K$. Notice that $*_{\sigma}=\psi(c)+*_{\sigma}$ eo where $\sigma_{e o} \in \Gamma_{e o}(R)$ because $\Gamma_{c}(R) \perp \Gamma_{e o}^{*}(R)$ and $\psi(c) \in \Gamma_{c}(R)$ is unique by the Riesz representation theorem. Hence

$$
\left(\omega^{\prime}, \psi^{\prime}\left(c^{\prime}\right)\right)_{R^{\prime}}=\int_{c} f_{n}^{\#}\left(\omega^{\prime}\right)=\left(f_{n}^{\#}\left(\omega^{\prime}\right), *^{*}\right)_{K^{\circ}}
$$

Because $\left\|f_{n}^{\#}\left(\omega^{\prime}\right)-f^{\#}(\omega)\right\|_{K} \rightarrow 0$, by Proposition 6 it follows that

$$
\begin{aligned}
\left(\omega^{\prime}, \psi^{\prime}\left(c^{\prime}\right)\right)_{R^{\prime}} & =\left(f^{\#}\left(\omega^{\prime}\right),{ }^{*} \sigma\right)_{K}=\left(f^{\#}\left(\omega^{\prime}\right),{ }^{*} \sigma\right)_{R} \\
& =\left(f^{\#}\left(\omega^{\prime}\right), \psi(c)\right)_{R},
\end{aligned}
$$

which is the desired result.

This now permits us to show that $f^{\#}$ is an isomorphism of $\Gamma_{s e}\left(R^{\prime}\right)$ onto $\Gamma_{s e}(R)$. Let $d$ be any dividing cycle on $R$ and $\omega^{\prime} \in \Gamma_{s e}\left(R^{\prime}\right)$, then from Theorem 4, $0=\left(\omega^{\prime}, \psi^{\prime}\left(d^{\prime}\right)\right)_{R^{\prime}}=\left(f^{\sharp}\left(\omega^{\prime}\right), \psi(d)\right)_{R}$ because $\Gamma_{c}\left(R^{\prime}\right)=\Gamma_{\text {se }}\left(R^{\prime}\right) \oplus \Gamma_{b m}^{*}\left(R^{\prime}\right)$ and $\psi^{\prime}\left(d^{\prime}\right) \in \Gamma_{b m}^{*}\left(R^{\prime}\right)$ if and only if $d^{\prime}$ is a dividing cycle [3, Theorem $20 \mathrm{E}$, p. 311]. Thus, $\left(f^{\#}\left(\omega^{\prime}\right), \psi(d)\right)_{R}=0$ for all dividing cycles $d$ on $R$. As $d$ runs through all dividing cycles, the $\psi(d)$ span $\Gamma_{b m}^{*}(R)[3$, Theorem 20C, p. 310]. Therefore, $\left(f^{\#}\left(\omega^{\prime}\right), \sigma\right)_{R}=0$ for all $\sigma \in \Gamma_{b m}^{*}(R)$. From $f^{\#}\left(\omega^{\prime}\right) \in \Gamma_{c}(R)$ and $\Gamma_{c}(R)=\Gamma_{s e}(R)$ $\oplus \Gamma_{b m}^{*}(R)$, we conclude that $f^{\#}\left(\omega^{\prime}\right) \in \Gamma_{s e}(R)$. At this point the proof of Theorem 4 is complete. 
Next, we derive one more basic fact about the isomorphism $f^{\#}$; we shall determine its norm. If $f: R \rightarrow R^{\prime}$ is $K$-quasiconformal; then Theorem 2(i) shows that the norm $\left\|f^{\#}\right\|$ of the isomorphism $f^{\#}: \Gamma\left(R^{\prime}\right) \rightarrow \Gamma(R)$ is at most $K^{1 / 2}$. The following theorem improves this result.

Theorem 5. Suppose that $f: R \rightarrow R^{\prime}$ is a quasiconformal bomeomorphism baving maximal dilatation $K(f)$ and $f^{\#}: \Gamma\left(R^{\prime}\right) \rightarrow \Gamma(R)$ is the induced isomorphism, then $\left\|f^{\#}\right\|^{2}=K(f)$.

Proof. Clearly, it is enough to show that $K(f) \leq\left\|f^{\#}\right\|^{2}$ since we have already established the reverse inequality. The idea for the following proof is derived from $[17,7 G$, Pp. 214-215].

Let $\mathfrak{Q}(R)$ denote the set of all ring domains $A$ on $R$ such that $A$ is contained in a noncompact simply connected region $\Omega_{A}$ on $R$ and such that the two boundary components of $A$ are closed Jordan curves. It is known that

$$
K(f)=\sup _{A \in \mathbb{Q}(R)} \frac{M(f(A))}{M(A)},
$$

where $M(A)$ denotes the modulus of the ring domain $A$. Given $A \in \mathbb{Q}(R)$ let $A_{0}$ denote the compact component of $R \sim A$ in $\Omega_{A}$. For each $A \in \mathbb{Q}(R)$ let $b_{A}$ be the unique continuous real-valued function on $R$ with the properties that $b_{A} \mid A_{0}$ $\equiv 1, b_{A} \mid R \sim\left(A \cup A_{0}\right) \equiv 0$ and $b_{A} \mid A$ is harmonic. It is not difficult to see that $d b_{A} \in \Gamma_{e}(R)$ and it is well known that $M(A)=\left\|d b_{A}\right\|_{R}^{-2}$.

Assume that $\left\|f^{\#}\right\|^{2}<K(f)$, then there is an $A \in \mathcal{Q}(R)$ such that

$$
\left\|f^{\#}\right\|^{2}<M(f(A)) / M(A) \leq K(f) \text {. }
$$

Because $M(A)=\left\|d b_{A}\right\|_{R}^{-2}$ and $M(f(A))=\left\|d b_{f(A)}\right\|_{R^{\prime}}^{-2}$, we obtain

$$
\left\|f^{\#}\right\|<\frac{\left\|d b_{A}\right\|_{R}}{\left\|d b_{f(A)}\right\|_{R}} \text {. }
$$

Observe that $f^{\#}\left(d b_{f(A)}\right)=d\left(b_{f(A)} \circ f\right) \in \Gamma_{e}(R)$ and that the continuous function $b_{f(A)} \circ f$ has the properties that $b_{f(A)} \circ f \mid A_{0} \equiv 1$ and $b_{f(A)} \circ f \mid R \sim\left(A \cup A_{0}\right) \equiv 0$. Hence, a suitable version of the Dirichlet principle implies that $\left\|d b_{A}\right\|_{R} \leq$ $\left\|f^{\#}\left(d b_{f(A)}\right)\right\|_{R}$, because $b_{f(A)}$ of has the same boundary values on $\partial A$ as the harmonic function $b_{A}$. Consequently,

$$
\left\|f^{\#}\right\|<\frac{\left\|f^{\#}\left(d h_{f(A)}\right)\right\|_{R}}{\left\|d b_{f(A)}\right\|_{R^{\prime}}},
$$

which contradicts the definition of the norm of $f^{\#}$. This establishes the theorem.

Corollary. The isomorphism $f^{\#}: \Gamma\left(R^{\prime}\right) \rightarrow \Gamma(R)$ is an isometry if and only if the quasiconformal mapping $f: R \rightarrow R^{\prime}$ is actually a conformal mapping. 
Proof. If $f^{\#}$ is an isometry, then $\left\|f^{\#}\right\|=1$. Thus, $K(f)=1$ and $f$ is a conformal mapping. Conversely, if $f$ is conformal, then elementary computations show that $f^{\#}$ is an isometry.

3. Isomorphism of square integrable harmonic differentials induced by a quasiconformal mapping. Marden $[6, p .30]$ has shown that it is possible to use a quasiconformal map $f: R \rightarrow R^{\prime}$ to define an isomorphism of $\Gamma_{b}\left(R^{\prime}\right)$ onto $\Gamma_{b}(R)$. Of course, for a general quasiconformal mapping $f: R \rightarrow R^{\prime}$ and $\omega^{\prime} \in \Gamma_{b}\left(R^{\prime}\right)$, one cannot expect that $f^{\#}\left(\omega^{\prime}\right) \in \Gamma_{b}(R)$. However, $f^{\#}\left(\omega^{\prime}\right) \in \Gamma_{c}(R)$ and $\Gamma_{c}^{b}(R)=\Gamma_{b}(R)$ $\oplus \Gamma_{e 0}(R)$ so one can take the projection of $f^{\#}\left(\omega^{\prime}\right)$ onto the subspace $\Gamma_{b}(R)$; let $f_{b}^{\#}\left(\omega^{\prime}\right)$ denote the image of $f^{\#}\left(\omega^{\prime}\right)$ under this projection. The following analog of Theorem 2 is valid.

Theorem 6. (i) If $f: R \rightarrow R^{\prime}$ is $K$-quasiconformal, then

$$
f_{b}^{\#}\left(a \omega^{\prime}+b \sigma^{\prime}\right)=a f_{b}^{\#}\left(\omega^{\prime}\right)+b f_{b}^{\#}\left(\sigma^{\prime}\right)
$$

for all $a, b \in \mathbf{C}$ and $\omega^{\prime}, \sigma^{\prime} \in \Gamma_{b}\left(R^{\prime}\right)$ and

$$
K^{-1 / 2}\left\|\omega^{\prime}\right\|_{R^{\prime}} \leq\left\|f_{b}^{\#}\left(\omega^{\prime}\right)\right\|_{R} \leq K^{1 / 2}\left\|\omega^{\prime}\right\|_{R^{\prime}}
$$

(ii) If $j: R \rightarrow R$ is the identity mapping, then $j_{b}^{\#}: \Gamma_{b}(R) \rightarrow \Gamma_{b}(R)$ is the identity mapping.

(iii) If $f: R \rightarrow R^{\prime}$ and $g: R^{\prime} \rightarrow R^{\prime \prime}$ are quasiconformal bomeomorphisms, then $(g \circ f)_{b}^{\#}=f_{b}^{\#} \circ g_{b}^{\#}$.

Proof. We shall only verify (iii) since the other parts are apparent. Let $\omega^{\prime \prime} \epsilon$ $\Gamma_{b}\left(R^{\prime \prime}\right)$, then $g^{\sharp}\left(\omega^{\prime \prime}\right)=g_{b}^{\sharp}\left(\omega^{\prime \prime}\right)+\omega_{e o}^{\prime}$ where $\omega_{\text {eo }}^{\prime} \in \Gamma_{e o}\left(R^{\prime}\right)$. Hence,

$$
(g \circ f)^{\#}\left(\omega^{\prime \prime}\right)=f^{\#}\left(g^{\#}\left(\omega^{\prime \prime}\right)\right)=f^{\#}\left(g_{b}^{\#}\left(\omega^{\prime \prime}\right)\right)+f^{\#}\left(\omega_{e o}^{\prime}\right) .
$$

Now, $f^{\#}\left(\omega_{e o}^{\prime}\right) \in \Gamma_{e o}(R)$ since $f^{\#}$ is an isomorphism of $\Gamma_{e o}\left(R^{\prime}\right)$ onto $\Gamma_{e o}(R)$. Also, $f^{\#}\left(g_{b}^{\#}\left(\omega^{\prime \prime}\right)\right)=f_{b}^{\#}\left(g_{b}^{\#}\left(\omega^{\prime \prime}\right)\right)+\omega_{e o}$ where $\omega_{e o} \in \Gamma_{e o}(R)$. Therefore

$$
(g \circ f)^{\#}\left(\omega^{\prime \prime}\right)=f_{b}^{\#}\left(g_{b}^{\#}\left(\omega^{\prime \prime}\right)\right)+\omega_{e o}+f^{\#}\left(\omega_{e o}^{\prime}\right) .
$$

Since $f_{b}^{\#}\left(g_{b}^{\#}\left(\omega^{\prime \prime}\right)\right) \in \Gamma_{b}(R)$ and $\omega_{e o}+f^{\#}\left(\omega_{e o}^{\prime}\right) \in \Gamma_{e o}(R)$, the uniqueness of the orthogonal decomposition implies that $(g \circ f)_{b}^{\#}=f_{b}^{\#} \circ g_{b}^{\#}$.

Corollary. If $f: R \rightarrow R^{\prime}$ is quasiconformal, then $f_{b}^{\#}: \Gamma_{b}\left(R^{\prime}\right) \rightarrow \Gamma_{b}(R)$ is an isomorphism of Hilbert spaces.

There is also a counterpart to Theorem 3. 
Theorem 7. If $f: R \rightarrow R^{\prime}$ is a quasiconformal bomeomorphism, then the isomorpbism $f_{b}^{\#}: \Gamma_{b}\left(R^{\prime}\right) \rightarrow \Gamma_{b}(R)$ is also an isomorphism of $\Gamma_{b s e}\left(R^{\prime}\right), \Gamma_{b e}\left(R^{\prime}\right)$, $\Gamma_{b o}\left(R^{\prime}\right), \Gamma_{b m}\left(R^{\prime}\right)$ onto $\Gamma_{b s e}(R), \Gamma_{b e}(R), \Gamma_{b o}(R), \Gamma_{b m}(R)$, respectively.

Proof. The result for $\Gamma_{b o}$ is already known $\left[6\right.$, p. 30]. Only the fact that $f_{b}^{\#}$ is an isomorphism of $\Gamma_{b s e}\left(R^{\prime}\right)$ onto $\Gamma_{b s e}(R)$ will be established in detail since all of the remaining cases can be handled in the same manner. Let $\omega^{\prime} \in \Gamma_{b s e}\left(R^{\prime}\right)$ $C \Gamma_{s e}\left(R^{\prime}\right)$, then $f^{\#}\left(\omega^{\prime}\right) \in \Gamma_{\text {se }}(R)$ because $f^{\#}$ is an isomorphism of $\Gamma_{s e}\left(R^{\prime}\right)$ onto $\Gamma_{s e}(R)$. Now, $f^{\#}\left(\omega^{\prime}\right)=f_{b}^{\#}\left(\omega^{\prime}\right)+\omega_{e o}$ where $\omega_{e o} \in \Gamma_{e o}(R) \subset \Gamma_{\text {se }}(R)$. Hence, $f_{b}^{\#}\left(\omega^{\prime}\right)=f^{\#}\left(\omega^{\prime}\right)-\omega_{e o} \in \Gamma_{s e}(R) \cap \Gamma_{b}(R)=\Gamma_{b s e}(R)$. This proves that $f_{b}^{\#}$ maps $\Gamma_{b s e}\left(R^{\prime}\right)$ into $\Gamma_{b s e}(R)$. By consideration of $f^{-1}$ we can conclude that $f_{b}^{\#}$ is surjective. The other results follow in the same manner by making use of the facts that $f^{\#}$ is an isomorphism of $\Gamma_{e}\left(R^{\prime}\right), \Gamma_{c o}\left(R^{\prime}\right), \Gamma_{c o e}\left(R^{\prime}\right)$ onto $\Gamma_{e}(R), \Gamma_{c o}(R)$, $\Gamma_{c o e}(R)$, respectively, that $\Gamma_{e}(R), \Gamma_{c o}(R), \Gamma_{c o e}(R) \supset \Gamma_{e o}(R)$ and that $\Gamma_{e}(R)$ $\cap \Gamma_{b}(R)=\Gamma_{b e}(R), \Gamma_{c o}(R) \cap \Gamma_{b}(R)=\Gamma_{b o}(R), \Gamma_{c o e}(R) \cap \Gamma_{b}(R)=\Gamma_{b m}(R)$.

Thus, Theorem 7 shows that the isomorphism $f_{b}^{\#}$ preserves many of the important subspaces of harmonic differentials; however, not all such subspaces are preserved. For instance, let $\Gamma_{a e}\left(R^{\prime}\right)$ denote the subspace of exact analytic differentials and $\Gamma_{S}\left(R^{\prime}\right)$ the subspace of Schottky differentials on $R^{\prime}$, then it need not be true that $f_{b}^{\sharp}\left(\Gamma_{a e}\left(R^{\prime}\right)\right)=\Gamma_{a e}(R)$ or $f_{b}^{\#}\left(\Gamma_{S}\left(R^{\prime}\right)\right)=\Gamma_{S}(R)$. By definition $R^{\prime} \in O_{A D}$ if and only if $\Gamma_{a e}\left(R^{\prime}\right)=(0)$, and $\Gamma_{b}\left(R^{\prime}\right)=\Gamma_{S}\left(R^{\prime}\right)$ if and only if $R^{\prime} \in O_{A D}$ [3, Corollary 14E, p. 294]. The class $O_{A D}$ is not quasiconformally invariant [17, Theorem 14A, p. 135], so there is a quasiconformal homeomorphism $f: R \rightarrow R^{\prime}$ of Riemann surfaces such that $R \notin O_{A D}$ and $R^{\prime} \in O_{A D^{*}}$. Therefore, $f_{b}^{\#}\left(\Gamma_{a e}\left(R^{\prime}\right)\right)=$ (0) is a proper subset of $\Gamma_{a e}(R)$. Also, from $\Gamma_{b}\left(R^{\prime}\right)=\Gamma_{S}\left(R^{\prime}\right)$ and $\Gamma_{b}(R) \neq \Gamma_{S}(R)$, we see that $f_{b}^{\#}\left(\Gamma_{S}\left(R^{\prime}\right)\right)=\Gamma_{b}(R)$ properly contains $\Gamma_{S}(R)$. Consequently, the isomorphism of harmonic differentials which is induced by a quasiconformal mapping need not always preserve the space of Schottky differentials or the space of exact analytic differentials.

This same result can be used to demonstrate that an additional subspace of harmonic differentials is not preserved. It is known that $\Gamma_{s}(R)=$ $\mathrm{Cl}\left(\Gamma_{b o}(R)+\Gamma_{b o}^{*}(R)\right)[3$, Theorem 14D, p. 293]. From this it is readily seen that the subspace $\Gamma_{b o}^{*}$ is generally not preserved. Suppose that $f_{b}^{\#}\left(\Gamma_{b o}^{*}\left(R^{\prime}\right)\right)=\Gamma_{b o}^{*}(R)$, then $f_{b}^{\#}\left(\Gamma_{b o}\left(R^{\prime}\right)+\Gamma_{b o}^{*}\left(R^{\prime}\right)\right)=\Gamma_{b o}(R)+\Gamma_{b o}^{*}(R)$ because we already know that $f_{b}^{\#}\left(\Gamma_{b o}\left(R^{\prime}\right)\right)=\Gamma_{b o}(R)$. As $f_{b}^{\#}$ is an isomorphism, we may now conclude that $f_{b}^{\sharp}\left(\Gamma_{S}\left(R^{\prime}\right)\right)=\Gamma_{S}(R)$, a result not always true.

The fact that $\Gamma_{b o}^{*}$ is not preserved by $f_{b}^{\#}$ is at odds with a result of Marden [6, Theorem 14, p. 31]. Let us restate part of his theorem in our notation so that we can see how it implies the invariance of $\Gamma_{b o}^{*}$ under the isomorphism $f_{b}^{\#}$. Let $f: R \rightarrow R^{\prime}$ be a quasiconformal homeomorphism, $c$ a compact 1-cycle on $R$ and 
$c^{\prime}=f(c)$. Suppose $\psi(c) \in \Gamma_{b o}^{*}(R)$ is the $\Gamma_{b}(R)$-reproducer for $c$ and $\psi^{\prime}\left(c^{\prime}\right) \epsilon$ $\Gamma_{b o}^{*}\left(R^{\prime}\right)$ is the $\Gamma_{b}\left(R^{\prime}\right)$-reproducer for $c^{\prime}$, then one part of Marden's theorem asserts that

$$
f_{h}^{\#}\left(\psi^{\prime}\left(c^{\prime}\right)\right)=\frac{\left\|\psi^{\prime}\left(c^{\prime}\right)\right\|_{R^{\prime}}}{\left\|g_{b}^{\#}(\psi(c))\right\|_{R^{\prime}}} \psi(c),
$$

where $g=f^{-1}$. In other words, for every compact cycle $c^{\prime}$ on $R^{\prime}, f_{b}^{\#}\left(\psi^{\prime}\left(c^{\prime}\right)\right)$ is a scalar multiple of $\psi(c)$. In particular, $f_{b}^{\#}\left(\psi^{\prime}\left(c^{\prime}\right)\right) \in \Gamma_{b o}^{*}(R)$ for all compact 1-cycles $c^{\prime}$ on $R^{\prime}$. Now, as $c^{\prime}$ runs through the set of all compact cycles on $R^{\prime}$, the corresponding set of all such reproducing differentials $\psi^{\prime}\left(c^{\prime}\right)$ span $\Gamma_{b o}^{*}\left(R^{\prime}\right)[3$, Theorem 20C, p. 310]. From this we can conclude that $f_{b}^{\#}\left(\Gamma_{b o}^{*}\left(R^{\prime}\right)\right) \subset \Gamma_{h o}^{*}(R)$. Due to the symmetry of the situation, equality must hold, or $f_{b}^{\#}$ preserves $\Gamma_{b o}^{*}$, a result which we know to be generally false.

The preceding indicates the invalidity of the theorem of Marden; it seems worthwhile to isolate the error in his proof. In order to achieve this, it is necessary to paraphrase his proof. Suppose $L$ is the linear functional on $\Gamma_{b}(R)$ determined by $c$ so that $L(\omega)=\int_{\mathfrak{c}} \omega=(\omega, \psi(\mathrm{c}))_{R}$ for all $\omega \in \Gamma_{b}(R)$, and let $L^{\prime}$ be the associated linear functional defined on $\Gamma_{b}\left(R^{\prime}\right): L^{\prime}\left(\omega^{\prime}\right)=L\left(f_{b}^{\#}\left(\omega^{\prime}\right)\right)$. Previously, we have shown that $L^{\prime}\left(\omega^{\prime}\right)=\int_{c^{\prime}} \omega^{\prime}=\left(\omega^{\prime}, \psi^{\prime}\left(c^{\prime}\right)\right)_{R^{\prime}}$, where $c^{\prime}=f(c)$. Marden's result is that $f_{b}^{\#}$ maps $\psi^{\prime}\left(c^{\prime}\right)$ into a scalar multiple of $\psi(c)$. One key identity in his "proof" is

$$
\max _{\omega^{\prime} \in I^{\prime}}\left(\omega^{\prime}, \psi^{\prime}(c)\right)_{R^{\prime}}=\max _{\omega^{\prime} \in I^{\prime}}\left(f_{b}^{\#}\left(\omega^{\prime}\right), \psi(c)\right)_{R}=\max _{\omega \in I}(\omega, \psi(c))_{R^{\prime}},
$$

where $I^{\prime}=\left\{\omega^{\prime} \in \Gamma_{b}\left(R^{\prime}\right):\left\|\omega^{\prime}\right\|_{R^{\prime}}=1\right\}$ is the unit sphere in $\Gamma_{b}\left(R^{\prime}\right)$ and $I=f_{b}^{\#}\left(I^{\prime}\right)=$ $\left\{\omega \in \Gamma_{b}(R):\left\|g_{b}^{\#}(\omega)\right\|_{R^{\prime}}=1\right\}$. His "proof" hinges on the fact that the only differential in $I$ for which $\max \omega \in I,(\omega, \psi(c))_{R}$ is attained is $\psi(c) /\left\|g_{b}^{\#}(\psi(c))\right\|_{R}$. However, this is generally not the differential for which the maximum is obtained.

In this area there are still several open questions. At present it is not known whether any of the classes $\Gamma_{b s e}^{*}, \Gamma_{b e}^{*}$ and $\Gamma_{b m}^{*}$ is preserved by $f_{b}^{\#}$ in the general case. They are trivially preserved when $f$ is a conformal mapping since it is readily verified that $f^{\#}(\omega)=* f^{\#}(\omega)$ in this case and we know that $\Gamma_{b s e}, \Gamma_{b e}$ and $\Gamma_{b m}$ are preserved. Also, what is the norm of $f_{b}^{\#}$ ?

4. Related work. In this section we shall discuss related work of Accola, Nakai and Rochberg; connections and similarities between their work and $\$ \$ 2$ and 3 will be presented.

In a series of papers Nakai ([7], [8], [9], [10], [11]) investigated the correspondence between certain algebraic isomorphisms and quasiconformal mappings; a complete exposition may be found in [17, Chapter III]. We restate his main result in a form suitable for our purposes; it is equivalent to his original version. Given 
a Riemann surface $R$ let $M(R)$ denote the Royden algebra associated with $R ; M(R)$ is the set of all bounded Tonelli functions defined on $R$ having finite Dirichlet integral. $M(R)$ is a Banach algebra relative to the norm $\|b\|=\sup _{R}|b|+\|d b\|_{R}$. It is clear that $d M(R)=\{d b: b \in M(R)\} \subset \Gamma_{e}(R)$. A quasiconformal mapping $f:$ $R \rightarrow R^{\prime}$ determines an algebraic isomorphism $f^{b}: M\left(R^{\prime}\right) \rightarrow M(R)$ defined by $f^{b}(b)$ $=b \circ f$. Conversely, an algebra isomorphism $\sigma: M\left(R^{\prime}\right) \rightarrow M(R)$ induces a quasiconformal map $f: R \rightarrow R^{\prime}$ such that $\sigma=f^{b}$. Thus, the algebraic structure of Royden's algebra $M(R)$ characterizes the quasiconformal structure of the Riemann surface $R$.

It is elementary to see that $d f^{b}(b)=f^{\#}(d b)$ for $b \in M\left(R^{\prime}\right)$ so that the isomorphisms $f^{\#}$ and $f^{b}$ are intimately connected. This also shows that $f^{\#}$ maps $d M\left(R^{\prime}\right)$ onto $d M(R)$. An obvious question to consider is the extent to which an analog of the work of Nakai holds for the correspondence between quasiconformal maps and isomorphisms of Hilbert spaces of differential forms. As we have seen, every quasiconformal mapping $f: R \rightarrow R^{\prime}$ induces an isomorphism $f^{\#}: \Gamma\left(R^{\prime}\right) \rightarrow$ $\Gamma(R)$ and $\left\|f^{\#}\right\|=K(f)^{1 / 2}$. Conversely, is every isomorphism of $\Gamma\left(R^{\prime}\right)$ onto $\Gamma(R)$ induced by some quasiconformal map of $R$ onto $R^{\prime}$ ? The answer to this question is negative. Given two quasiconformally equivalent Riemann surfaces $R$ and $R^{\prime}$, the coefficient of quasiconformality of $R$ with respect to $R^{\prime}$ is defined to be $K\left(R, R^{\prime}\right)=\inf K(f)$, where the infimum is taken over all quasiconformal homeomorphisms $f: R \rightarrow R^{\prime}$. Note that $K\left(R, R^{\prime}\right) \geq 1$. Clearly, a necessary condition for an isomorphism $\phi: \Gamma\left(R^{\prime}\right) \rightarrow \Gamma(R)$ to be determined by some quasiconformal map $f: R \rightarrow R^{\prime}$; that is, for $\phi=f^{\#}$, is $\|\phi\| \geq K\left(R, R^{\prime}\right)$. However, this condition is certainly not sufficient. For any Riemann surface $R, \Gamma(R)$ is a separable Hilbert space of countably infinite orthogonal dimension. Consequently, for any two Riemann surfaces $R$ and $R^{\prime}, \Gamma(R)$ and $\Gamma\left(R^{\prime}\right)$ are isometric, so the Hilbert space structure of $\Gamma(R)$ does not determine the quasiconformal structure of $R$. It would be interesting to determine a reasonable characterization of those isomorphisms which are induced by a quasiconformal mapping.

Rochberg ([13], [14]) has shown how to define a distance between two compact bordered Riemann surfaces. For a compact bordered Riemann surface $\bar{R}$, let $A(\bar{R})$ be the supremum normed Banach algebra of functions continuous on $\bar{R}$ and holomorphic on $R$, the interior of $\bar{R}$. Given two compact bordered Riemann surfaces $\bar{R}_{1}$ and $\bar{R}_{2}$, the distance between them is defined by making use of the family of all continuous invertible linear maps from $A\left(\bar{R}_{1}\right)$ to $A\left(\bar{R}_{2}\right)$. This idea can be carried out in the setting of this paper. Given two Riemann surfaces $R$ and $R^{\prime}$, let $L\left(\Gamma(R), \Gamma\left(R^{\prime}\right)\right)$ denote the set of all continuous linear isomorphisms of $\Gamma(R)$ onto $\Gamma\left(R^{\prime}\right)$. Given $\phi \in L\left(\Gamma(R), \Gamma\left(R^{\prime}\right)\right)$, set $c(\phi)=\left(\|\phi\|\left\|\phi^{-1}\right\|\right)^{-1}$ and $d\left(R, R^{\prime}\right)=\inf \left\{-\log c(\phi): \phi \in L\left(\Gamma(R), \Gamma\left(R^{\prime}\right)\right)\right\}$. With this definition $d\left(R, R^{\prime}\right)=0$ since $\Gamma(R)$ and $\Gamma\left(R^{\prime}\right)$ are isometric. We could try to restrict our attention to $L\left(\Gamma_{b}(R), \Gamma_{b}\left(R^{\prime}\right)\right)$. But if $R$ and $R^{\prime}$ are compact Riemann surfaces of the same 
genus, then $\Gamma_{b}(R), \Gamma_{b}\left(R^{\prime}\right)$ have the same dimension and are isometric. Thus, $R$ and $R^{\prime}$ would have zero distance between them. This program would meet with more success if those isomorphisms induced by a quasiconformal map could be characterized in a natural manner and then we replaced $L\left(\Gamma(R), \Gamma\left(R^{\prime}\right)\right)$ by this smaller family.

Accola [2] has shown how to induce an isomorphism of a certain subspace of harmonic differentials from a mapping more general than a quasiconformal mapping. We recall the construction.

Definition. Let $f: R \rightarrow R^{\prime}$ be a homeomorphism of Riemann surfaces. Given a cycle $c$ on $R$, let $c^{\prime}$ be the corresponding cycle on $R^{\prime}$. Let $\mathcal{H}(c), \mathcal{H}\left(c^{\prime}\right)$ denote the class of all cycles on $R, R^{\prime}$ which are homologous to $c, c^{\prime}$, respectively. $f$ is said to be an bo-mapping if there is a finite constant $M \geq 1$ such that

$$
M^{-1} \lambda(\mathcal{H}(\mathrm{c})) \leq \lambda\left(\mathcal{H}\left(\mathrm{c}^{\prime}\right)\right) \leq M \lambda(\mathcal{H}(\mathrm{c}))
$$

for all cycles $c$ on $R$, where $\lambda(\mathcal{H})$ denotes the extremal length of the family $\mathcal{H}$. If $f: R \rightarrow R^{\prime}$ is an bo-mapping, then $R^{\prime}$ is said to be bo-equivalent to $R$.

Clearly, $f: R \rightarrow R^{\prime}$ is an bo-mapping if and only if $f^{-1}$ is. Also, a quasiconformal map is an bo-map. The converse is not necessarily true: any homeomorphism of the unit disk onto the complex plane is an bo-mapping but these two regions are not quasiconformally equivalent.

It was demonstrated by Accola [2, Theorem 2, p. 541] that an bo-mapping $f: R \rightarrow R^{\prime}$ induces an isomorphism $f^{*}$ of $\Gamma_{b o}^{*}\left(R^{\prime}\right)$ onto $\Gamma_{b o}^{*}(R)$. The mapping is defined by $f^{*}\left(\psi^{\prime}\left(c^{\prime}\right)\right)=\psi(c)$ for corresponding cycles $c$ and $c^{\prime}$; recall that $\psi(c)$

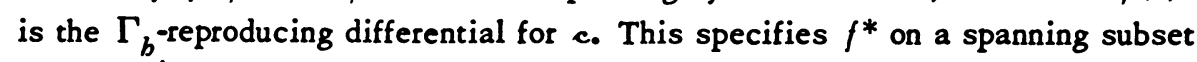
of $\Gamma_{b o}^{*}\left(R^{\prime}\right)$. The fact that $f$ is an bo-mapping implies that $f^{*}$ is continuous, so $f^{*}$ extends to all of $\Gamma_{b o}^{*}\left(R^{\prime}\right)$. A sort of converse is true. If $f: R \rightarrow R^{\prime}$ is a homeomorphism and $\phi: \Gamma_{b o}^{*}\left(R^{\prime}\right) \rightarrow \Gamma_{b o}^{*}(R)$ is a continuous linear isomorphism such that $\phi\left(\psi^{\prime}\left(c^{\prime}\right)\right)=\psi(c)$ for all corresponding cycles $c$, $c^{\prime}$, then $f$ is an bo-mapping. We establish the following property of $f^{*}$ which we shall use in the next section.

Theorem 8. If $f: R \rightarrow R^{\prime}$ is an bo-mapping, then $f^{*}\left(\Gamma_{b m}^{*}\left(R^{\prime}\right)\right)=\Gamma_{b m}^{*}(R)$.

Proof. As $d^{\prime}$ runs through all dividing cycles on $R^{\prime}$, the differentials $\psi^{\prime}\left(d^{\prime}\right)$ span $\Gamma_{b m}^{*}\left(R^{\prime}\right)$ [3, Theorem 20C, p. 310]. Moreover, $d^{\prime}$ is a dividing cycle if and only if $d$ is. These two comments together with the definition of $f^{*}$ prove the theorem.

What is the connection between $f^{*}$ and $f_{b}^{\#}$ ? Recall that $f_{b}^{\#}$ generally does not preserve $\Gamma_{b \circ}^{*}$. If $f: R \rightarrow R^{\prime}$ is a conformal mapping, then $f^{*}=f_{b}^{\#} \mid \Gamma_{b 0^{*}}^{*}$ It is not known whether equality holds under any other conditions. 
5. Classification theory of Riemann surfaces. Several results in the classification theory of Riemann surfaces can be obtained by making use of various properties of the isomorphisms $f^{\#}$ and $f^{*}$. Let $n$ be a positive integer; a Riemann surface $R$ is said to be of class $O_{H D}^{n}, O_{K D}^{n}, O_{H M}^{n}$ if $\Gamma_{b e}(R), \Gamma_{b e}(R) \cap \Gamma_{b s e}^{*}(R)$, $\Gamma_{b m}(R)$, respectively, has dimension $n-1$. In particular, $O_{H D}=O_{H D}^{1}$; similarly for $O_{K D}$ and $O_{H M}$.

Theorem 9. (i) The classes $O_{H D}^{n}$ and $O_{K D}^{n}$ are preserved under quasiconformal equivalence.

(ii) The class $O_{H M}^{n}$ is preserved under bo-equivalence.

Proof. (i) From Theorem 7, if $f: R \rightarrow R^{\prime}$ is a quasiconformal homeomorphism, then $f_{b}^{\#}$ maps $\Gamma_{b e}\left(R^{\prime}\right)$ onto $\Gamma_{b e}(R)$. Thus, they both have the same dimension.

Recall that $\Gamma_{b e}=\Gamma_{b m} \oplus\left(\Gamma_{b e} \cap \Gamma_{b s e}^{*}\right)$, so that $\Gamma_{b e} / \Gamma_{b m} \cong \Gamma_{b e} \cap \Gamma_{b s e^{*}}^{*}$ Since $f_{b}^{\#}$ is an isomorphism of $\Gamma_{b e}\left(R^{\prime}\right)$ onto $\Gamma_{b e}(R)$ which maps $\Gamma_{b m}\left(R^{\prime}\right)$ onto $\Gamma_{b m}(R)$, it follows that $\Gamma_{b e}\left(R^{\prime}\right) / \Gamma_{b m}\left(R^{\prime}\right)$ is isomorphic to $\Gamma_{b e}(R) / \Gamma_{b m}(R)$. In particular, $\Gamma_{b e}\left(R^{\prime}\right) \cap \Gamma_{b s e}^{*}\left(R^{\prime}\right)$ and $\Gamma_{b e}(R) \cap \Gamma_{b s e}^{*}(R)$ have the same dimension.

(ii) This is a simple consequence of Theorem 8 since $f^{*}$ is an isomorphism of $\Gamma_{b m}^{*}\left(R^{\prime}\right)$ onto $\Gamma_{b m}^{*}(R)$.

That the classes $O_{H D}^{n}$ and $O_{K D}$ are quasiconformally invariant is known [17, Theorem 14B, p. 135 and Theorem $8 \mathrm{H}$, p. 221]; the other results seem to be new. Our final result is to prove that belonging to the class $O_{H M}$ is a property of the ideal boundary.

Theorem 10. Belonging to the class $O_{H M}$ is a property of the ideal boundary.

Proof. Let $R$ and $S$ be Riemann surfaces and take bordered boundary neighborhoods $A$ and $B$ on $R$ and $S$, respectively. Thus, $A^{\prime}=R \sim A$ and $B^{\prime}=R \sim B$ are regular subregions of $R$ and $S$, respectively. Consider a conformal homeomorphism $f$ of $A$ onto $B$; we assume that $f$ is defined on $\partial A$ and maps it onto $\partial B$. Suppose that $R \in O_{H M}$; we want to show that $S \in O_{H M}$. Let $R^{*}$ and $S^{*}$ denote the Kerékjártó-Stoïlow compactification of $R$ and $S$, respectively. Take $\beta_{1}$ and $\beta_{2}$ to be two closed disjoint subsets of the ideal boundary of $S$. Because $f$ induces a homeomorphism of $R^{*} \sim A^{\prime}$ onto $S^{*} \sim B^{\prime}$, there exist corresponding disjoint closed sets $\alpha_{1}$ and $\alpha_{2}$ on the ideal boundary of $R^{*} . R \in O_{H M}$ is equivalent to the statement that there do not exist two disjoint closed subsets of ideal boundary components with positive capacity [15, Theorem 1C, p. 202]. Therefore, we may assume that $a_{1}$ has capacity zero. Necessary and sufficient for $a_{1}$ to have capacity zero is that the family of arcs in $R^{*}$ joining $\partial A$ to $\alpha_{1}$ have infinite extremal length [15, Theorem 4B, Pp. 165-166]. This is the same as saying that the family of arcs in $R^{*} \sim A^{\prime}$ joining $\partial A$ to $\alpha_{1}$ has infinite extremal length. Since extremal 
length is a conformal invariant, the family of curves in $S^{*} \sim B^{\prime}$ connecting $\partial B$ to $\beta_{1}$ also has infinite extremal length. Consequently, $\beta_{1}$ has capacity zero and it follows that $S \in O_{H M}$ since there do not exist two disjoint closed subsets of the ideal boundary of $S$ having positive capacity.

\section{REFERENCES}

1. R. D. M. Accola, The bilinear relation on open Riemann surfaces, Trans. Amer. Math. Soc. 96 (1960), 143-161. MR 23 \#A2521.

2. - Differentials and extremal length on Riemann surfaces, Proc. Nat. Acad. Sci. U. S. A. 46 (1960), 540-543. MR 22 \#9598.

3. L. V. Ahlfors and L. Sario, Riemann surfaces, Princeton Math. Series, no. 26, Princeton Univ. Press, Princeton, N. J., 1960. MR 22 \#5729.

4. I. Kra, Automorphic forms and Kleinian groups, Benjamin, New York, 1972.

5. O. Lehto and K. I. Virtanen, Quasikonforme Abbildungen, Die Grundlehren der math. Wissenschaften in Einzeldarstellungen mit besonderer Berücksichtigung der Anwendungsgebiete, Band 126, Springer-Verlag, Berlin and New York, 1965. MR 32 \#5872.

6. A. Marden, The weakly reproducing differentials on open Riemann surfaces, Ann. Acad. Sci. Fenn. Ser. AI No. 359 (1965), 32pp. MR 32 \#5868.

7. M. Nakai, On a ring isomorphism induced by quasiconformal mappings, Nagoya Math. J. 14 (1959), 201-221. MR 21 \#1379.

8. - A function algebra on Riemann surfaces, Nagoya Math. J. 15 (1959), 1-7. MR $21 \# 5726$.

9. - Purely algebraic characterization of quasiconformality, Proc. Japan Acad. 35 (1959), 440-443. MR $22 \# 3813$.

10. - On a problem of Royden on quasiconformal equivalence of Riemann surfaces, Proc. Japan Acad. 36 (1960), 33-37. MR 22 \#2691.

11. - Algebraic criterion on quasiconformal equivalence of Riemann surfaces, Nagoya Math. J. 16 (1960), 157-184. MR 22 \#1669.

12. A. Pfluger, Theorie der Riemannschen Flächen, Die Grundlehren der math. Wissenschaften, Band 89, Springer-Verlag, Berlin, 1957. MR 18, 796.

13. R. Rochberg, Almost isometries of Banach spaces and moduli of planar domains (to appear).

14. - Almost isometries of Banach spaces and moduli of Riemann surfaces, Duke Math. J. 40 (1973), 41-52.

15. B. Rodin and L. Sario, Principal functions, Van Nostrand, Princeton, N. J., 1968. MR 37 \#378.

16. H. L. Royden, On a class of null-bounded Riemann surfaces, Comment. Math. Helv. 34 (1960), 52-66. MR 22 \#1663.

17. L. Sario and M. Nakai, Classification theory of Riemann surfaces, Die Grundlehren der math. Wissenschaften, Band 164, Springer-Verlag, New York and Berlin, 1970. MR 41 \#660.

18. J. Väisälä, Lectures on n-dimensional quasiconformal mappings, Lecture Notes in Math., no. 229, Springer-Verlag, New York, 1971.

DEPARTMENT OF MATHEMATICS, UNIVERSITY OF CINCINNATI, CINCINNATI, OHIO 45221 